\title{
Application of multi regressive linear model and neural network for wear prediction of grinding mill liners
}

\author{
Farzaneh Ahmadzadeh \\ Division of operation and maintenance \\ Luleå University of Technology \\ Luleå, Sweden
}

\author{
Jan Lundberg \\ Division of operation and maintenance \\ Luleå University of Technology \\ Luleå, Sweden
}

\begin{abstract}
The liner of an ore grinding mill is a critical component in the grinding process, necessary for both high metal recovery and shell protection. From an economic point of view, it is important to keep mill liners in operation as long as possible, minimising the downtime for maintenance or repair. Therefore, predicting their wear is crucial. This paper tests different methods of predicting wear in the context of remaining height and remaining life of the liners. The key concern is to make decisions on replacement and maintenance without stopping the mill for extra inspection as this leads to financial savings. The paper applies linear multiple regression and artificial neural networks (ANN) techniques to determine the most suitable methodology for predicting wear. The advantages of the ANN model over the traditional approach of multiple regression analysis include its high accuracy.
\end{abstract}

Keywords-Wear prediction; Remaining useful life; Artificial neural network; Principal Component Analysis; Maintenance scheduling ; Condition Monitoring.

\section{INTRODUCTION}

The development of a maintenance system for mechanical structures that has both intelligent features in fault detection and knowledge accumulation is an academic goal for researchers as it can greatly assist industry where it is now almost impossible to manually analyse the rapidly growing data to extract valuable decision-making information.

Engineering prognostics is used by industry to manage business risks that result from equipment failing unexpectedly; reliability estimation of equipment and estimation of its remaining useful life (RUL) are mandatory [1].

In practice, such estimations remain predominantly intuitive and are based on the experience of personnel familiar with the equipment. However, due to improved asset reliability and an ageing engineering workforce, it is increasingly difficult to rely on experience. Furthermore, human decision making is not always sufficiently reliable or accurate when dealing with complex equipment with a multitude of interrelated failure modes.

Therefore, developing methods to reduce industry's dependence on human experience is desirable. Appropriate model selection to ensure successful practical implementation requires a mathematical understanding of each model type and an appreciation of how a particular business intends to utilise the models and their outputs. In reality, industry sites will not be able to use every prognostic modelling option with equal efficacy. The models' ability to perform the modelling is highly dependent on the availability of required data, skilled personnel and computing infrastructure. Consequently, model requirements must be clearly understood [2].

For example, autogenous mills used in the mining industry and in ore dressing plants can cause major bottlenecks in downtime and negatively influence production economics. The rubber liners inside these mills are critical for protecting the mill shell and grinding ore. The replacement and inspection of these mill liners are major factors in mill stoppages and lead to production losses. Therefore, the wear prediction of mill liners is critical for making replacement decisions, as is prediction accuracy.

Selecting the appropriate wear prediction method can lead to a significant reduction in the overall costs $[3,4]$. This paper examines two methodologies, linear multiple regression and artificial neural networks (ANN), to determine which is best for prediction [5].



Fig. 1. Cross sectional view of grinding mill and Shell feed lifter bar (by Metso Mineral)

\section{DATA COLLECTION AND PREPARATION}

Assessing wear using life cycle data is hampered because of the unavailability of operating information, particularly in the wear out phase of liner measurement. The data requirements 
for this research were met by selecting the shell feed lifter bar (LB) of the liner of grinding mill machines see Fig. 1. The following sources were used to gather life cycle and condition monitoring $(\mathrm{CM})$ data.

- Metso mineral $\mathrm{AB}$ for wear measurement data during different life cycles.

- Boliden mineral $\mathrm{AB}$ for process data during the same life cycle.

Process data for five years were obtained from the Boliden mining company. The process data include the ore type, ore feed $(\mathrm{ton} / \mathrm{h})$, power $(\mathrm{kW})$, angular speed (\% of centrifugal critical speed), torque ( $\%$ of the max torque), water addition $\left(\mathrm{m}^{3} / \mathrm{h}\right)$, grinding energy $(\mathrm{kWh} / \mathrm{ton})$, load (ton). The mill in this case study processes ore types which come from different mines and have different physical characteristics in grade values (\% of metal content), densities, hardness indexes, rock size etc.

\section{A. Interpolation Technique}

Because total value of lost production during any mill stoppage is extremely high, it is not economical to stop the mill at intervals and measure liner wear, except for maintenance, inspection, installation, and replacement. As a result, few wear measurement data were available from Metso. The solid circles and triangle in Fig. 2 show real measured remaining height and remaining life of LB during one life cycle; other data shown in the figure were generated by piecewise cubic Hermit interpolating polynomial (PCHIP). PCHIP preserves the shape of the data and respects monotonicity. It is the best interpolating method for this study because of the monotonically deceasing characteristics of CM data in the context of the remaining height and remaining life of the liners.

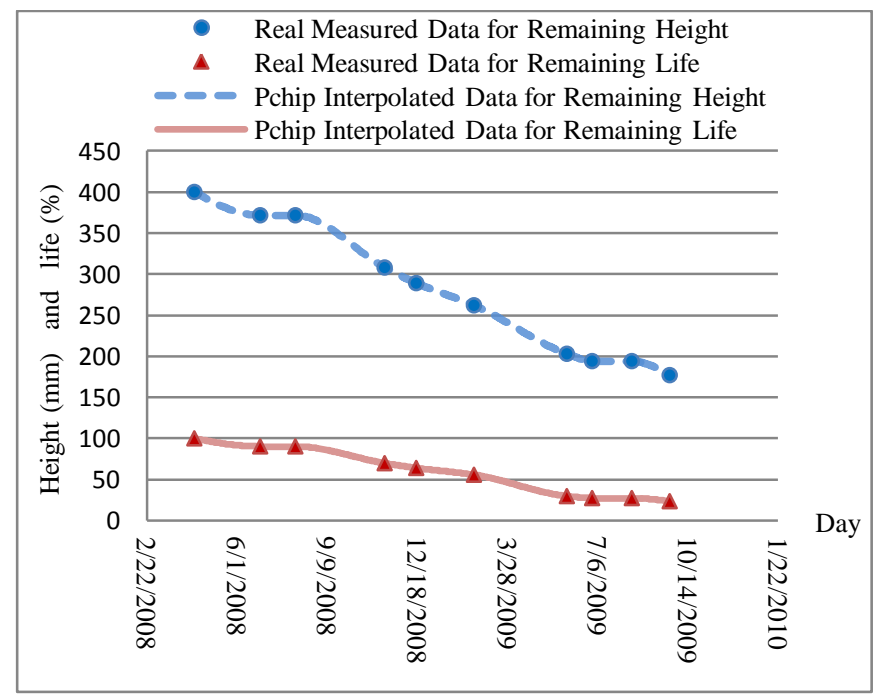

Real and interpolated data using PCHIP method

Methodologies

Making reliable decisions on maintenance and replacement of the liners' components requires a thorough analysis of their behaviour during their life cycle. As every product has a different operating environment, individual assessment of the capacity of each component is necessary.

$\mathrm{CM}$ and process data must be analysed to identify which parameters can be used to estimate the wear of components. As recommended in $[5,6]$, the following methods were selected for analysing and investigating these data to determine the wear of components under given conditions of use.

\section{B. Multiple Regression Analysis}

Regression analysis is one of the most useful tools to estimate and forecast future trends of variables by analysing historical data. It is an extremely flexible procedure that can aid decision making in many areas, such as sales, medicine, weather forecasting etc. Regression is a technique used to predict the value of a dependent variable using one or more independent variables. Mathematically,

$$
Y=a+b_{1} x_{1}+b_{2} x_{2}+\ldots+b_{k} x_{k}
$$

Where $Y$ is the response variable, $a$ and $b_{i} i=1 \ldots k$ are regression coefficients, $\mathrm{x}_{1}, \mathrm{x}_{2} \ldots \ldots \mathrm{x}_{\mathrm{k}}$ represent the explanatory variables, and $\mathrm{K}$ is the total number of explanatory variables.

The method of least squares estimation as [7] is used to calculate the regression coefficients. R-square, the coefficient of determination is used as the performance measure to determine how successfully the method explains the variation of the data [8]. However, the purpose of carrying out regression analysis is to know how the explanatory variables, also called predictors or independent variables, such as ore type, ore feed, power, speed, torque, water addition, grinding energy, and load, are related to the response (dependent) variables in this case, the remaining height and remaining life of the LB. In other words, the primary goal is to estimate or predict the LB's wear given current and past values of the explanatory variables.

The regression analysis is done using Microsoft Excel. It can perform stepwise regression analysis that helps to determine the impact of each of the explanatory variables in the system. The multiple regression equation can be expressed as (2). Applying a multiple regression technique to the data yields the following regression equation:

$$
\begin{aligned}
& \text { Wear }=a+b_{1}(\text { Oretype })+b_{2}(\text { Orefeed })+b_{3}(\text { Power })+b_{4}(\text { Speed })+\cdots \\
& b_{5}(\text { Torque })+b_{6}(\text { Water })+b_{7}(\text { energy })+b_{8}(\text { Load })
\end{aligned}
$$

Where, wear in (2) shows the remaining height or remaining life of the liner. The multiple regression coefficients' p-values and the correlation results for when the dependent variable is either the remaining life or the remaining height of the LB for shell feed are shown in Tables I and II respectively. 
TABLE I.

Multiple REgRESSION ANALYSis RESUlts WHEN DEPENDENT VARIABLE (WEAR) Is REMAINING LiFE

\begin{tabular}{|c|c|c|c|c|c|c|c|c|c|c|}
\hline Wear & Intercept & Ore type & Ore feed & Power & Speed & Torque & Load & $\begin{array}{c}\text { Grinding } \\
\text { energy }\end{array}$ & $\begin{array}{c}\text { Water } \\
\text { addition }\end{array}$ & $\mathbf{R}^{2}$ \\
\hline Coefficients & 298,77 & 1,44 & $-0,04$ & 0,17 & $-4,12$ & $-3,32$ & 0,01 & $-0,43$ & 3,13 & 0,40 \\
\hline P-value & $0 \%$ & $2 \%$ & $0 \%$ & $3 \%$ & $3 \%$ & $1 \%$ & $98 \%$ & $82 \%$ & $0 \%$ & \\
\hline
\end{tabular}

TABLE II.

MultiPle Regression ANALysis Results When DEPENDENT VARIABLE (WEAR) Is REMAINING HeIGHT

\begin{tabular}{|c|c|c|c|c|c|c|c|c|c|c|}
\hline Wear & Intercept & Ore type & Ore feed & Power & Speed & Torque & Load & $\begin{array}{c}\begin{array}{c}\text { Grinding } \\
\text { energy }\end{array} \\
\end{array}$ & $\begin{array}{c}\begin{array}{c}\text { Water } \\
\text { addition }\end{array} \\
\end{array}$ & $\mathbf{R}^{2}$ \\
\hline P-value & $0 \%$ & $1 \%$ & $0 \%$ & $3 \%$ & $3 \%$ & $1 \%$ & $86 \%$ & $76 \%$ & $0 \%$ & 0,42 \\
\hline
\end{tabular}

TABLE III.

MultiPle REgRESSION ANALYSIS RESUlts WHEN DEPENDENT VARIABLE (WEAR) Is REMAINING LIFE AFTER APPLYING STEPWISE REGRESSION

\begin{tabular}{|c|c|c|c|c|c|c|c|c|}
\hline Wear & Intercept & Ore type & Ore feed & Power & Speed & Torque & $\begin{array}{c}\text { Water } \\
\text { addition }\end{array}$ & $\mathbf{R}^{2}$ \\
\hline Coefficients & 308,29 & 1,42 & $-0,03$ & 0,18 & $-4,37$ & $-3,47$ & 3,10 \\
\hline P-value & $0 \%$ & $2 \%$ & $0 \%$ & $2 \%$ & $1 \%$ & $0 \%$ & $0 \%$ \\
\hline
\end{tabular}

TABLE IV. MULTIPLE REgRESSION ANALYSIS RESULTS WHEN DEPENDENT VARIABLE (WEAR) IS REMAINING LIFE AFTER APPLYING STEPWISE REGRESSION

\begin{tabular}{|c|c|c|c|c|c|c|c|c|}
\hline Wear & Intercept & Ore type & Ore feed & Power & Speed & Torque & $\begin{array}{c}\text { Water } \\
\text { addition }\end{array}$ & $\mathbf{R}^{2}$ \\
\hline Coefficients & 1039,04 & 4,19 & $-0,09$ & 0,53 & $-12,84$ & $-10,84$ & 8,78 \\
\hline P-value & $0 \%$ & $1 \%$ & $0 \%$ & $1 \%$ & $1 \%$ & $0 \%$ & $0 \%$ \\
\hline
\end{tabular}

Because we want an explanatory model, we only keep variables where the error ( $\mathrm{p}$-value) is less than 0.05 , giving us a 95\% confidence level. Stepwise regression (backward elimination) is used; this involves starting with all candidate variables, testing the deletion of each variable (those with a pvalue greater than $5 \%$ ) to determine whether this improves the model, and repeating this process until no further improvement is possible.

In Tables I and II, the load and grinding energy which have p-values greater than 5\% are deleted from (1); this does not improve the $\mathrm{R}^{2}$ but the rest of variables are significant at $95 \%$ confidence level; see results in Tables III and IV. Thus, the remaining height and remaining life of the shell feed LB can be predicted, following (3), (4), as

Re main_life $=a+b_{1}($ Oretype $)+b_{2}($ Orefeed $)+b_{3}($ Speed $)+\cdots$

$b_{4}($ Torque $)+b_{5}($ Water $)$

Remain_hight $=a+b_{1}($ Oretype $)+b_{2}($ Orefeed $)+\cdots$

$b_{3}($ Power $)+b_{4}$ (Torque $)+b_{5}($ Water $)$

Despite this remarkable improvement in establishing a good correlation between input and output variables, the multiple regression results are still not acceptable for decision making on life assessment because of the low value of the coefficient of determination, $\left(\mathrm{R}^{2}\right)$. In the best case scenario, fitted models for the remaining height and remaining life explain 40 and $42 \%$ respectively of the total variation in the data.

\section{Artificial Neural Network}

Artificial neural networks (ANNs) are a special case of adaptive networks; they have been extensively explored in the literature because they can perform nonlinear modelling without a priori knowledge and are able to learn complex relationships among inputs and outputs. Moreover, from a computational point of view, ANNs are quick processes. The idea of using ANNs for forecasting is not new. For example, [9] used the windrow's adaptive linear network to forecast the weather. However, due to the lack of a training algorithm at the time, the research was limited and ANNs were left aside. Since the 1980s, research has expanded. One of the first successful applications of ANNs in forecasting is reported by [10] who designed a feed forward ANN to accurately mimic a chaotic series. In general, feed forward ANNs (PMC, RBF) trained with the back propagation algorithm have been found to perform better than classical autoregressive models for trend prediction in nonlinear time series [11, 12]. Many factors can affect the performance of ANNs (number of inputs and outputs nodes, number of layers, activation functions, learning algorithm, training sample etc.). Thus, building a neural network predictor is a non-trivial task. Since the 1990s, many studies have sought to improve the accuracy of predictions while reducing the time required for processing. ANNs have successfully been used to support the prediction process, and research work emphasizes its importance. Nevertheless, some authors remain skeptical, feeling that the design of an ANN is more of an art than a science, or calling ANNs black boxes, implying that an ANN has no explicit form to explain or analyze the relationships between inputs and outputs. However, 
ANNs have been used to find nonlinear or linear relationships between input (process data) and output (CM) variables, thereby predicting the values for CM data which show wear. The proposed ANN model has the following characteristics:

1) Architecture: The proposed neural network model is a multilayer feed-forward back-propagation neural network introduced in [13]; see Fig. 3. The back-propagation neural network model has the advantages of handling non-linear problems with a learning capability [14]. The architecture of the proposed network consists of two hidden layers of sigmoid (tansig) neurons followed by an output layer of a linear neuron (purelin). Hidden layers with nonlinear transfer functions allow the network to learn nonlinear and linear relationships between input and output variables, while the linear transfer function of the output layer lets the network produce outputs outside the range $[-1,1]$.

The number of inputs to the proposed network is given by the number of available inputs or process data (ore type, ore feed $(\mathrm{ton} / \mathrm{h})$, power $(\mathrm{kW})$, speed $(\%)$, torque $(\%)$, water addition $\left(\mathrm{m}^{3} / \mathrm{h}\right)$, grinding energy ( $\left.\mathrm{kWh} / \mathrm{ton}\right)$, and load (ton)); the number of neurons in the output layer is constrained to two, remaining height and remaining life.

The number and size of layers between network inputs and the output layer were determined by testing several combinations of numbers of layers and various numbers of neurons in each layer. Each of the selected combinations was tested with several different initial conditions to guarantee that the proposed model could provide the best solution. The resulting network consists of two hidden layers of 25 and 50 neurons respectively. See Fig. 3 for its architecture.



Fig. 2. Architecture of the proposed ANN.

2) Data preparation: 886 data sets were collected. Among these data sets, $80 \%$ of the data (708 sets) were used for the neural networks' training phase, while the remaining 20\% (177 sets) were used to test the network. The testing data were grouped in multiples of 6: 6,12,18, and so on.

3) Training the network: The training style was supervised learning which provides a set of examples (the training set) of proper network behaviour. A training set consists of inputs and the corresponding correct outputs (targets). One of the most powerful learning algorithms, the Levenberg-Marquardt algorithm [13], was used to train the network. In function approximation problems, this algorithm is considered to have the fastest convergence.

4) Learning and generalization: After the training was completed, the network was tested for its learning and generalization capabilities. The test of its learning ability was conducted by testing its ability to produce outputs for the set of inputs (seen data) used in the training phase. The test for the network's generalization ability was carried out by investigating its ability to respond to the input sets (unseen data) that were not included in the training process.

Figs. 4 to 7 show relative error for predicted remaining height and remaining life in the training and testing phase (seen and unseen data). These indicate the proposed network's performance. As shown in Figs. 4 and 5 the maximum relative error was less than $6 \%$ and $10 \%$ for remaining height and life respectively for seen data during the training phase and less than $4 \%$ and $10 \%$ for remaining height and life respectively for unseen data during the testing phase.

In short, the network predicts the height and remaining life of the liners with accuracy greater than $90 \%$. Therefore, the proposed model can approximate the input-output function with high accuracy.

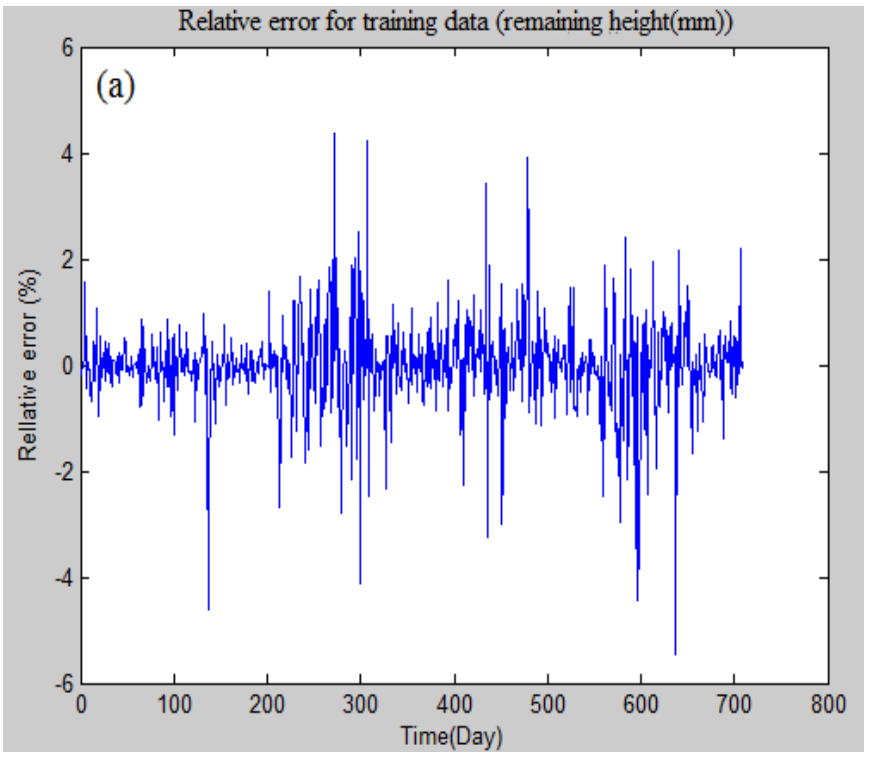

Fig. 3. Relative error for predicted remaining height for training phase 


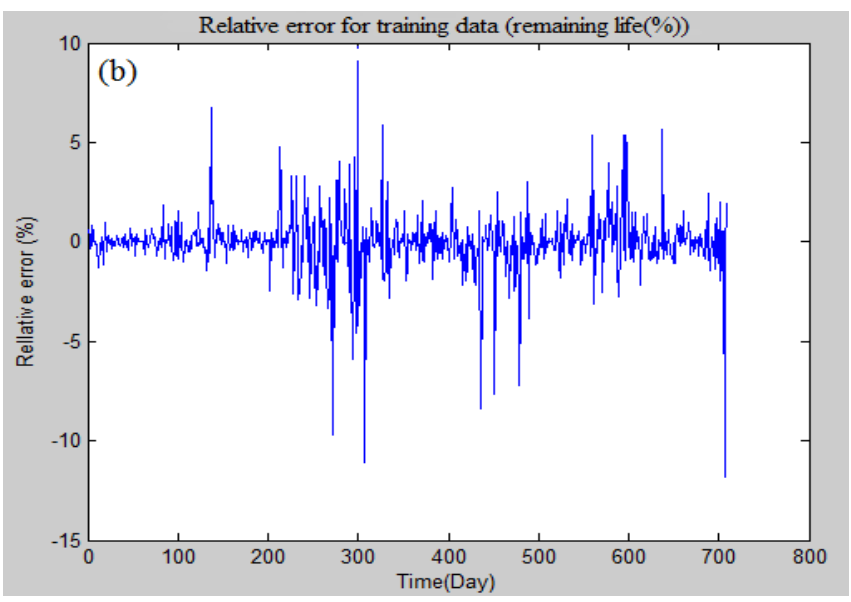

Fig. 4. Relative error for predicted remaining life for training phase

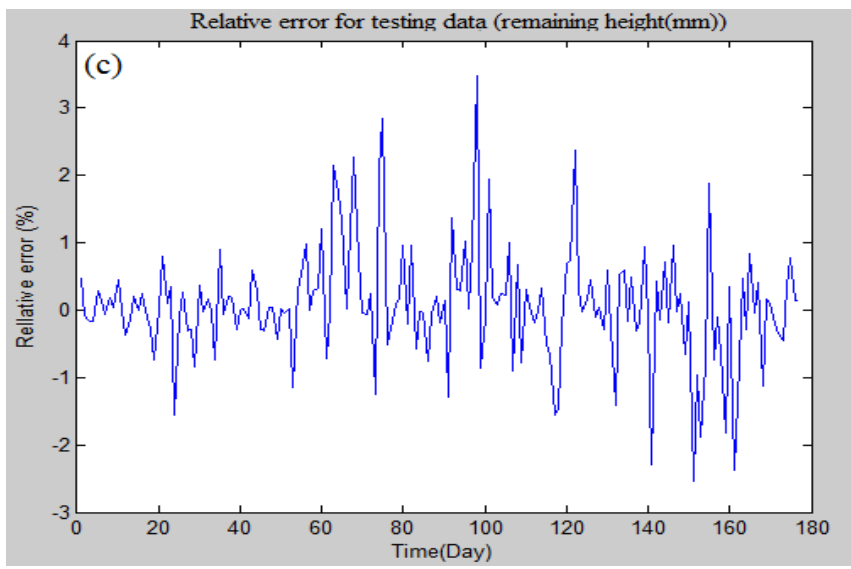

Fig. 5. Relative error for predicted remaining height for testing phase

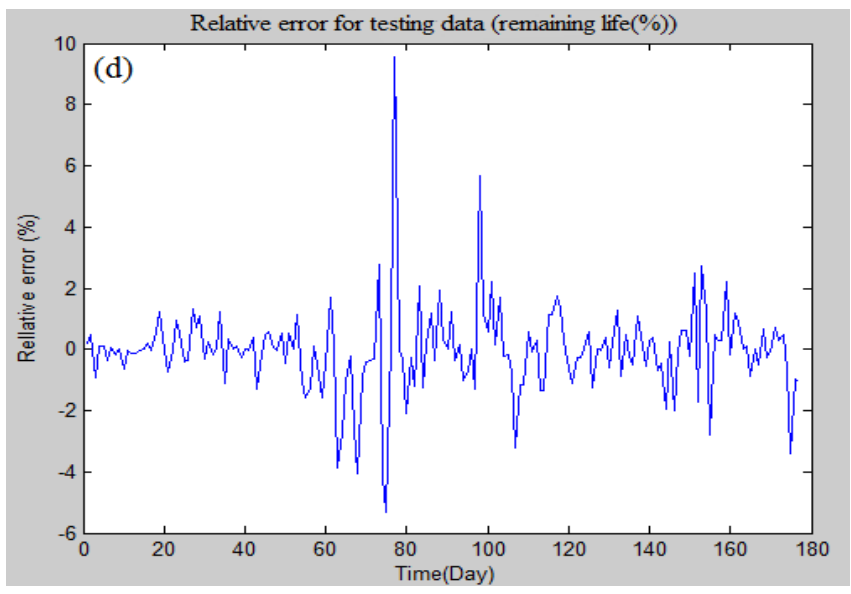

Fig. 6. Relative error for predicted remaining life for testing phase

5) Network performance: The performance of the neural network model is very consistent for both the training and testing data. The network's outputs have a correlation coefficient of about 0.99987 with the desired (actual) outputs, as shown in Fig. 8. Clearly, the neural network model is capable of handling the complex nonlinear interrelationships between variables. In addition, there was no substantial difference in network's output when it was trained with seen or unseen data.

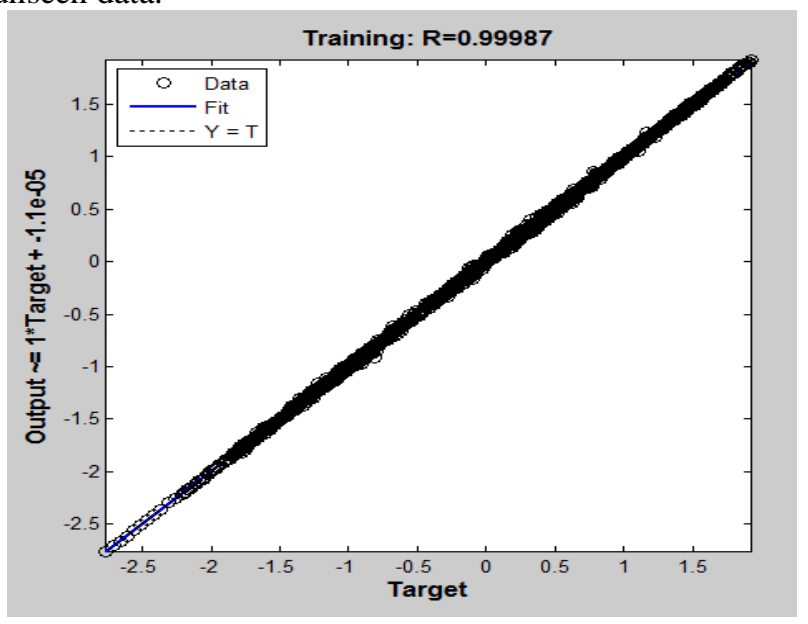

Fig. 7. Neural Network's Performance

\section{RESULTS AND DISCUSSION}

In this study, regression analysis and artificial neural networks were employed to develop input-output relationships for wear prediction. When we consider two life cycles of data (around five years), it is evident that based on the coefficients of determination ( $\mathrm{R}^{2}$ values), the artificial neural network model is the best of the two methods.

In one hand, regression analysis methods produce reasonable results in situations where the input variables follow a well-defined trend over the age of the component, but this method has been found struggling to maintain its estimation accuracy when the input variables exhibit a complex trend. This limits the application of regression procedures to restricted ranges of data sets making them unsuitable for the whole range of life cycle data. On the other hand, ANN which has been widely used for various prediction and forecasting problems is predominantly useful for many complex real-world problems because of their flexible nonlinear modeling ability.

A summary of the results appears in Table V. The results reveal that the classical procedures of regression analysis fails to produce acceptable results, as the correlation between input and output variables is very low. Further, the multiple regression analysis lacks the required level of accuracy, as the $\mathrm{R}^{2}$ is around $40 \%$ and $42 \%$ for remaining height and remaining life respectively, so it is not trustworthy for making decision for maintenance scheduling or replacement. But the proposed neural network can adapt to the data presented to it in the form of input-output patterns with high accuracy more than $99 \%$.

The model shown in Table $V$ has very high values of $\mathrm{R}^{2}$ for ANN. A comparison summary given in the table shows that the classical methods are no longer capable of producing reasonable results in situations where input-output relationships are nonlinear and complex. Once trained, however, the neural network model yields outputs very close to the desired targets. Therefore, the artificial neural network is the best methodology for wear prediction of grinding mill liners. 
TABLE V.

COMPARISON OF ESTIMATION ACCURACY FOR ANN AND REGRESSION MODEL

\begin{tabular}{|c|c|c|}
\hline \multirow{2}{*}{$\begin{array}{c}\text { Wear prediction } \\
\text { accuracy base on } \\
\text { method }\end{array}$} & \multicolumn{2}{|c|}{$\mathbf{R}^{2}$} \\
\cline { 2 - 3 } & $A N N$ & Multiple Regression \\
\hline Height & \multirow{2}{*}{0.99987} & 0.42 \\
\hline Life (Volume) & & 0.40 \\
\hline
\end{tabular}

IV. CONCLUSION

Wear prediction with nonlinear inputs is far more complicated than with linear inputs, especially in the case of an intricate mixture of fluctuating and unpredictable trends. This study has analysed life cycle data (inputs) by employing regression analysis and ANN to predict the wear of grinding mill liners. It finds the classical procedures of regression analysis inadequate to handle nonlinear and complex inputoutput relationships in life cycle data, as the correlations between input and output variables established by these techniques are low. The neural network, on the other hand, is very effective in this respect. Furthermore, ANNs are found to have $90 \%$ accuracy, and the performance of the proposed model has consistent results for both training and testing data. The study further reveals that employing the proposed ANN as well as condition monitoring data analysis tools is the key factor in securing remaining life estimates associated with higher levels of certainty because maximum relative error was less than $6 \%$ for remaining height and less than $10 \%$ for remaining life in both training and testing phases.

The findings represent a critical advance in sustainable management of maintenance procedures in industry, especially for heavy duty equipment like grinding mill liners which must work constantly, since it allows for a better understanding of not only service requirements of mill liners but the remaining life of each liners component.

The other advantages of using ANN includes: It doesn't require very expensive and sophisticated equipment for data recording and analysis. The accuracy of the results are very high. The ANN model can accommodate a wide range of input variables with complex and nonlinear input trends/patterns. The proposed methodology does not require disassembly of the liners or stoppage of grinding mills to make decisions on replacement, inspection, installation and maintenance scheduling. The dynamic nature of the proposed methodology opens up future studies of wear prediction for different categories of liner component such as lifter shell, inner lifter bar, shell plate, feed end discharge end, Etc.

\section{ACKNOWLEDGMENT}

The authors would like to thank Boliden, Metso Mineral for supporting this research and permission to publish this article. Special appreciation is extended to the operating maintenance engineers Jan Burstedt (Boliden), Lars Furtenbach and Magnus Eriksson (Metso) for sharing their valuable experience, knowledge, and data to improve the paper.

\section{REFERENCES}

[1] M. El-Koujok, R. Gouriveau and N. Zerhouni, "From monitoring data to remaining useful life: an evolving approach including uncertainty," 34th SReDA Seminar and 2nd Joint ESReDA/ESRA Seminar, Spain. , 2008.

[2] J.Z. Sikorska, M. Hodkiewicz, L. Ma, "Prognostic modeling options for remaining useful life estimation by industry”. Mech. Syst. Signal. Pr. , vol. 25, pp. 1803-1836, 2011.

[3] A. R. Wijaya, "Multivariate analysis to predict the lifetime of liner due to wear," Annual Rep., Lulea University of technology, Sweden. 2010.

[4] R. Dandotiya, J. Lundberg, "Replacement decision model for mill liners”. J Quality In Maint.Eng. In press. 2011.

[5] Lucifredi, C. and M. R. Mazzieri. "Application of Multiregressive Linear Models, Dynamic Kriging Models and Neural Network Models to Predictive Maintenance of Hydroelectric Power Systems," Mech. Syst. Signal Pr., vol. 14, pp. 471 - 494, 2000.

[6] D.C. Montgomery, Design And Analysis of Experiments: Response Surface Method and Designs. John Wiley and Sons: New Jersey, 2005.

[7] H. J. Motulsky, A. Christopoulos, Fitting Models to Biological Data Using Linear and Nonlinear Regression: A Practical Guide to Curve Fitting. 1st ed., Oxford: Newyork, 2004.

[8] M. J. C. Hu, E. Halbert, "An adaptive data processing system for weather forecasting”. J Appl. Meteor., vol. 3, pp. 513-523, 1964.

[9] A.Lapedes, R. Farber, "Nonlinear signal processing using neural networks: prediction and system modeling," Tech . Rep. LA-UR872662, Los Alamos National Laboratory. 1987

[10] G. Zhang, B.E. Patuwo and M.Y. Hu. "Forecasting with artificial neural networks: the state of the art," Int. J Forecasting, vol. 14, pp. 35-62, 1998.

[11] R.C.M Yam, P.W. Tse, L. Li and P. Tu, "Intelligent predictive decision support system for condition-based maintenance", Int. J Adv. Manuf. Tech., vol. 17, pp. 383-391, 2001.

[12] S. Haykin, Neural networks: a comprehensive foundation. 2nd ed., Upper Saddle Rever, New Jersey: Prentice Hall, 1999.

[13] G. P. Zhang, M. Qi., "Neural network forecasting for seasonal and trend time series," Eur. J Oper. Res., vol. 160, pp. 501 - 514, 2005.

[14] M.I. Mazhar, S. Kara, H. Kaebernick, "Remaining life estimation of used components in consumer products: life cycle data analysis by Weibull and artificial neural networks," J Oper. Manag., vol. 25, pp. 1184-1193. 2007. 